\title{
Quantifying the Uncertainty in Measurements for MBAC
}

\author{
Anne Nevin, Peder J. Emstad, Yuming Jiang, and Guoqiang Hu \\ Centre for Quantifiable Quality of Service in Communication Systems (Q2S)*, \\ Norwegian University of Science and Technology (NTNU), Trondheim, Norway \\ \{anne.nevin, peder.emstad, yuming. jiang, guoqiang. hu\}@q2s.ntnu. no
}

\begin{abstract}
In Measurement Based Admission Control (MBAC), the decision of accepting or rejecting a new flow is based on measurements of the current traffic situation. An in-depth understanding of the measurement error and its uncertainty is vital for the design of a robust MBAC. In this work, we study the measured parameters used by the MBAC and characterize their error. Our work differs significantly from previous work in that we find how the uncertainty in the measurements varies with the length of the observation window.
\end{abstract}

Keywords: MBAC, QoS, Measurements, Error, Uncertainty, Gaussian distribution.

\section{Introduction}

Measurement Based Admission Control (MBAC) has long been recognized as a promising solution for providing statistical Quality of Service(QoS) guarantees in packet switched networks. An MBAC does not require an a priori source characterization that in many cases may be difficult or impossible to attain. Instead, MBAC uses measurements to capture the behavior of existing flows and uses this information together with some coarse knowledge of a new flow, when making an admission decision for the requesting flow.

Consider a system where all parameters of the flows are known. Given the proper characteristics of a flow and the available bandwidth $c$, it is possible to determine the maximum average rate the system can handle and at the same time minimize the QoS violation such as loss/delay probability [1. In the following this maximum average rate will be termed $u c$, where $c$ denotes the total capacity of the system and $u$, which is a number between $[0,1]$ is a tuning parameter which varies with the flow characteristics. Using the simplest MBAC algorithm, the measured sum MBAC algorithm [2], a new arriving flow, with a bandwidth requirement $\nu$, will be accepted by the MBAC if:

$$
\hat{E}[R]+\nu \leq u c .
$$

* "Centre for Quantifiable Quality of Service in Communication Systems, Centre of Excellence" appointed by The Research Council of Norway, funded by the Research Council, NTNU and UNINETT. http://www.q2s.ntnu.no 
$\hat{E}[R]$ in (10) is the measured average rate which includes statistical error 3 . Though much research has been done on determining $u c$ and an overview can be found from 2] and 4, less attention has been given to the added uncertainty due to the measurement process. This measurement error may cause a flow to be accepted in error which again may cause increased QoS violation beyond what is actually predicted by the MBAC algorithm. Thus, an in-depth understanding of the measurements themselves and how they are affected by the underlying traffic is vital for the design of a robust MBAC. Particularly, measurements are improved when they are taken over longer intervals. However, flows generally only stay in the system for a limited time. There is a tradeoff between the accuracy and the validity of measurements. We state the question: How long do we need to measure in order to accept a flow with a certain degree of accuracy? The issue of measurement error exists naturally in all MBAC schemes, which makes the question even more appealing.

The objective of this paper is to quantify the uncertainty of measurement and provide answer to the question. In the study, both correlation characteristics of flows and flow dynamics will be taken into account A proper setting of the length of the measurement period has been of general concern in the MBAC literature. Based on simulations, the only conclusion that can be drawn is that different settings are needed for different traffic scenarios. A deeper analytical understanding of the measurement process and its error has been sought in [5] and [6]. Our work differs significantly from previous work in that we find how the uncertainty in the measurements vary with the length of the observation window.

The reminder of the paper is organized as follows. In the next section, the system model with assumptions and a framework considering flow arrivals and departures is given. Then follows the method of obtaining the measurements in Section 3 before the estimation error is treated in detail in Section 4. Section 5 presents an analytical evaluation before the conclusion is given in Section 6

\section{System Model, Assumptions and Performance Measures}

Flows compete for a limited resource, which is a link with capacity $c$ controlled by the MBAC that makes its admission decision based on the average aggregate rate of admitted flows (11). The flows, link and MBAC together form the system.

At the rate level, it is assumed that each flow is a stationary rate process $X_{i}(t)$ described by its mean and auto covariance function and that these are known parameters. All flows are independent such that the aggregate rate $R(t)=\sum_{i=1}^{n} X_{i}(t)$. A mixing of flow classes will cause increased complexity for the MBAC algorithm and also the measurement process. To simplify, only the homogenous case where flows belong to the same class will be considered. With the knowledge of the mean aggregate rate of the individual flows $\xi$, the current average aggregate rate can be specified by the current number of flows. The maximum number of flows the system can handle is thus $n_{\max }=u c / \xi$. 
At the flow level the system is dynamic in the sense that flows arrive and leave after some time. It is assumed that new flows arrive according to a Poisson process with parameter $\lambda$. The number of flows in progress can then be regulated by varying the flow arrival rate and/or flow lifetime. The offered traffic at flow level is the Erlang load denoted by $A$. With the arrival rate of new flows $\lambda$ and the expected flow lifetime $E\left(T_{L}\right), A$ in terms of number of flows is then given by:

$$
A=\lambda \cdot E\left(T_{L}\right) .
$$

To be able to see the effect of flow dynamics on measurement error, we define a framework at flow level similar to what is in [7. A new arriving flow will be accepted by the MBAC with probability $q_{i}$ in state $i$, where $i$ is the number of flows in the system. It is assumed that new flows arrive according to a Poisson process with mean $1 / \lambda$, and that the flow lifetime $T_{L}$, also a Poisson process, has mean $1 / \mu$.

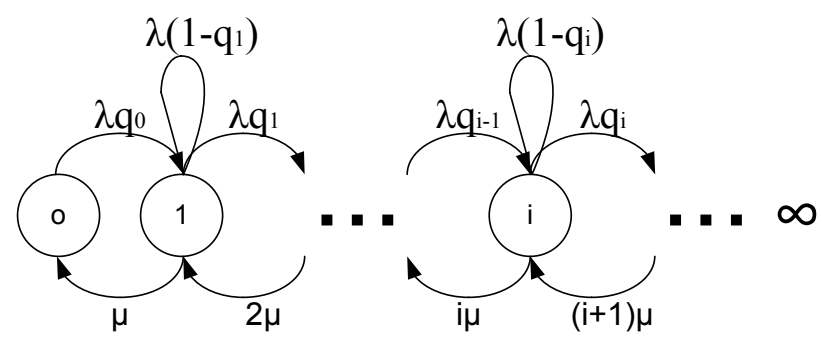

Fig. 1. State diagram of the number of sources accepted by the MBAC

Then the number of flows currently accepted by the MBAC follows a continuous-time Markov chain, see Fig. 1 and the probability, $P(i)$ of having $N=i$ flows in the system is:

$$
P(i)=\frac{\frac{A^{i}}{i !} \prod_{x=0}^{i-1} q_{x}}{\sum_{j=0}^{\infty} \frac{A^{j}}{j !} \prod_{x=0}^{j-1} q_{x}} .
$$

Implied by (3) and as also discussed in [7, the distribution $P(i)$ is indeed insensitive to the distribution of flow lifetime and only depends on the expected flow lifetime.

Upon the arrival of a flow, the decision process will make a correct or wrong admission decision. From a flow point of view, the performance measures related to the decision process are stated:

- Probability of false acceptance, denoted by $P_{F A c c}$, is defined as the probability of accepting a flow when it should have been rejected.

- Probability of false rejections, denoted by $P_{F R e j}$, is defined as the probability of rejecting a flow when it should have been accepted.

- Blocking Probability, denoted by $P_{B}$, is the overall blocking probability. 
With the continuous-time Markov system defined above, analytical expressions become:

$$
P_{F A c c}=\sum_{i=n_{\max }}^{\infty} P(i) \cdot q_{i}, P_{F R e j}=\sum_{i=1}^{n_{\max -1}} P(i) \cdot\left(1-q_{i}\right), P_{B}=\sum_{i=1}^{\infty}\left(1-q_{i}\right) P(i) .
$$

The $q_{i}$ s are yet to be determined and the reminder of the paper will give a deep insight into how the probability of accepting the requesting flow, i.e. $q_{i}$, is determined by the measurements. The way we shall use in determining $q_{i}$, where correlation between samples and the measurement errors are included, makes our framework significantly different from the work in [7.

\section{Measurement Process}

In this section, the method of obtaining the measured statistics will be given. The individual flow rate process $X(t)$ is observed every time slot $\Delta$, where $X_{i}$ is the observation at the end of time slot $i$. A measurement window $w$, consists of $m$ observations of the process, $w=m \Delta$. (See Fig. 2, where $R_{t}=\sum_{i}^{n} X_{i}(t)$ )

The rate process has mean $\xi$ and is assumed to be covariance stationary with covariance function $\rho(h)$ :

$$
\begin{aligned}
\rho(h) & =\operatorname{cov}\left(X_{i}, X_{i+h}\right) \\
& =E\left\{\left(X_{i}-E\left[X_{i}\right]\right)\left(X_{h+i}-E\left[X_{h+i}\right]\right)\right\} \\
& =E\left(X_{i} X_{h+i}\right)-\xi^{2} .
\end{aligned}
$$

\subsection{Measurement Method 1: Equidistant Sampling}

An instant measurement of the rate $X(t)$ is taken at every $t=\Delta i . X_{i}$ is the measured rate at the end of time slot $i$ given by $X_{i}=X\left(t_{i}\right)$. The measured sample $X=X_{1}, X_{2}, \ldots, X_{m}$ will be identically distributed but correlated observations, where the $X_{i} s$ have a sample mean, $\bar{X}$ given by

$$
\bar{X}=\frac{1}{m} \sum_{i=1}^{m} X_{i}
$$

A general expression for the variance of $\bar{X}, \operatorname{Var}(\bar{X})$, is given by $[8]$ :

$$
\begin{aligned}
\operatorname{Var}(\bar{X}) & \left.=E\left[(\bar{X}-\xi)^{2}\right)\right] \\
& =\frac{1}{m^{2}} \sum_{i=1}^{m} \sum_{j=1}^{m} E\left[\left(X_{i}-\xi\right)\left(X_{j}-\xi\right)\right],
\end{aligned}
$$

and with a covariance stationary process:

$$
\operatorname{Var}(\bar{X})=\frac{1}{m^{2}} \sum_{h=1-m}^{m-1}(m-|h|) \rho(h)
$$




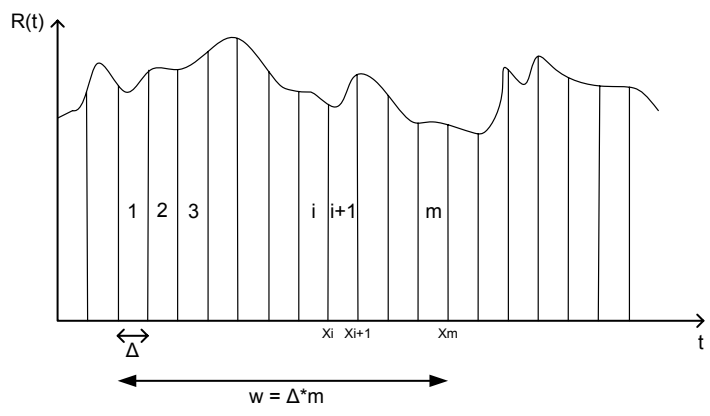

Fig. 2. Aggregate rate $\mathrm{R}(\mathrm{t})$ vs time

\subsection{Measurement Method 2: Continuous Observation}

The average rate over the window can also be found by continuous observation by letting the sampling rate go towards infinity. In practical sense this can be done by observing the time of change between busy and idle state on the incoming links. Let now $\Delta \rightarrow 0$ and $m \rightarrow \infty$ keeping the product $m \Delta$ constant such that $t_{i}=i \Delta \Rightarrow t$ then:

$$
\bar{X}=\frac{1}{m} \sum_{i=1}^{m} X_{i} \Rightarrow \lim _{\Delta \rightarrow 0 \mid w=m \Delta} \frac{1}{m} \sum_{i=1}^{m} X_{i}=\frac{1}{w} \int_{0}^{w} R(t) d t .
$$

Using limit considerations known from the literature, the time variance of the sample mean, $\zeta^{2}(w)$ can be found:

$$
\begin{aligned}
\zeta^{2}(w) & =\lim _{\Delta \rightarrow 0 \mid w=m \Delta} \operatorname{Var}(\bar{X}) \\
& =\lim _{\Delta \rightarrow 0 \mid w=m \Delta}\left(\frac{\Delta}{w}\right)^{2} \sum_{i=1-m}^{m-1}(m-|i|) \rho\left(t_{i}\right) \\
& =\frac{1}{w^{2}} \int_{-w}^{w}(w-|t|) \rho(t) d t \\
& =\frac{2}{w^{2}} \int_{0}^{w}(w-t) \rho(t) d t .
\end{aligned}
$$

Note that $\zeta^{2}(w)$ only depends on the window size and the auto-covariance function $\rho(t)$.

\section{Estimation Error}

In the following a detailed analysis of the estimation error will be presented to give an in-depth understanding of the accuracy of the measurement. A new flow is accepted based on measurements over a complete measurement window $w$ and 
we will assume that flows do not leave during this window. Each flow has a rate process $X_{i}(t)$ with mean $\xi$. The variance of the measured average rate of the flow rate process, $\zeta^{2}(w)$, given by (9), decreases as the window size increases.

With $N=n$ flows in the system,

$$
Y=\sum_{i=1}^{n} \frac{1}{w} \int_{0}^{w} X_{i}(t),
$$

is an estimator of the aggregate mean $n \xi$. According to the MBAC algorithm, (11) as long as $Y \leq \xi n_{\max }-\nu$ a new flow will be admitted. When $N=n_{\max }$, additional flows should be rejected but due to measurement error, underestimation of the aggregate rate will cause a flow to be admitted erroneously and constitutes a false acceptance. The probability of false acceptance for a flow $P_{F A c c}$ depends on the state probabilities and thus requires the inclusion of the reference system defined in Section 2. Here we will only consider a static system remaining in state $n_{\max }$ excluding the impact of flow dynamics. The requirement is to keep the probability of a false acceptance in state $n_{\max }$ below a performance target value $\varepsilon$ where typically $\varepsilon=0.05$. Conditioning on being in the state $n_{\max }$ the conditional performance target can be written:

$$
P\left(Y+\nu \leq \xi n_{\max } \mid N=n_{\max }\right) \leq \varepsilon .
$$

The probability of underestimating the aggregate mean increase as the measurement window size decreases. Because the measurement window size in general is very limited, it may be impossible to meet the required performance target given in (10). To cope with this problem, we reduce the maximum allowable bandwidth $\xi n_{\max }$ by a number of levels $l$ of size $\xi$, such that a new flow will only be accepted if:

$$
Y+\nu \leq \xi n_{\max }-l \xi, \quad l=0,1, \ldots n_{\max } .
$$

Dividing the bandwidth $n_{\max } \xi$ evenly between the flows, $\nu=\xi$ and the performance requirement is rewritten:

$$
P\left(Y+\xi \leq \xi n_{\max }-l \xi \mid N=n_{\max }\right) \leq \varepsilon, \quad l=0,1, \ldots n_{\max } .
$$

The task is now to determine $l$. This requires the distribution of $Y$ which can be found analytically for some simple sources. When the number of flows is large (say $n>30$ ), the sum of the time averages of the flows will be close to a normal distribution thus $Y \sim N\left(n \xi, n \zeta^{2}(w)\right)$. Conditioned on being in state $N=n_{\max }$ we have that:

$$
\left(\frac{Y-\xi n_{\max }}{\sqrt{n_{\max }} \zeta(w)} \leq z_{\varepsilon}\right)=1-\varepsilon,
$$

where $z_{\varepsilon}$ is the $\varepsilon-$ quantile, $F_{z}\left(z_{\varepsilon}\right)=1-\varepsilon$. Rearranging the terms gives:

$$
P\left(Y \leq \xi n_{\max }+\sqrt{n_{\max }} \zeta(w) z_{\varepsilon}\right)=1-\varepsilon
$$


and due to symmetry in the normal distribution:

$$
P\left(Y \leq \xi n_{\max }-\sqrt{n_{\max }} \zeta(w) z_{\varepsilon}\right)=\varepsilon .
$$

Comparing (15) and (12), the performance target (10) will be met if $l$ and $\zeta(w)$ satisfy:

$$
\xi(l+1)=\sqrt{n_{\max }} \zeta(w) z_{\varepsilon} .
$$

Since 1 is an integer, the requirement can be expressed:

$$
l+1=\left\lceil\frac{\sqrt{n_{\max }} \zeta(w) z_{\varepsilon}}{\xi}\right\rceil .
$$

For a given quantile and known $\zeta(w)$, this equation determines the required number of levels $l$ in the refined admission control algorithm (11).

With the introduction of levels, there will be a region between $n_{\max }$ and $n_{\max }-l$ where a flow may be admitted in error according to the condition given in (11) but will not necessarily be a false acceptance as defined in Section 2. We define the region between $n_{\max }$ and $n_{\max }-l$ the critical region and we define a Hazardous Admission to be the act of admitting a flow when the number of accepted flows is above $n_{\max }-l$ (See Fig. 3.).

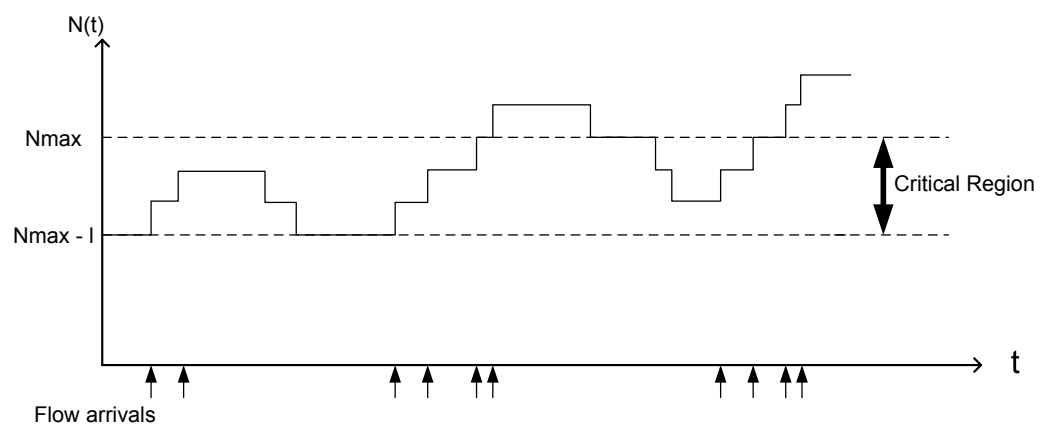

Fig. 3. Illustration of the critical region

Note again that (12) gives the probability of false acceptance conditioned on the system being in state $N=n_{\max }$. The probability of false acceptance for a flow $P_{F A c c}$ can only be found using the reference system defined in Section 2 . With the assumption of $Y \sim N\left(n \xi, n \zeta^{2}(w)\right)$ and $\nu=\xi$, the conditional accepting probability $q_{i}$ required in the reference system is written as:

$$
\begin{aligned}
q_{i} & =P(Y \leq u c-(l+1) \xi \mid N=i) \\
& =\frac{1}{\zeta(w) \sqrt{2 \pi i}} \int_{-\infty}^{u c-(l+1) \xi} e^{-\frac{(x-i \xi)^{2}}{2 i \zeta^{2}(w)}} d x,
\end{aligned}
$$


A study of the reference system will be addressed in a separate work where also dependence of the $q_{i}$ s at arrival is discussed.

\section{Case Study Using the MMRP Source Model}

In the following all flows belong to the same class and are of type Markov modulated rate process (MMRP), which is a process $X(t)=r I(t)$ where $\mathrm{I}(\mathrm{t})$ alternates between states $I=0$ and $I=1$. The duration of the 0 and 1 states follow a negative exponential distribution with mean $1 / \alpha$ and $1 / \beta$ respectively. Each flow is sampled by means of continuous observation. The auto-covariance of the MMRP process is given by:

$$
\operatorname{cov}\left(X_{i}, X_{i+\tau}\right)=\frac{r^{2} \alpha \beta}{(\alpha+\beta)^{2}} e^{-\tau(\alpha+\beta)} .
$$

Using (9) the expression for the variance of the sample mean is given by:

$$
\begin{aligned}
\zeta^{2}(w) & =\operatorname{var}(\bar{X})=\frac{2}{w^{2}} \int_{0}^{w}(w-t) \rho(t) d t \\
& =\frac{2 r^{2} \alpha \beta}{w^{2}(\alpha+\beta)^{3}}\left(w-\frac{1}{\alpha+\beta}\left(1-e^{-w(\alpha+\beta)}\right)\right) .
\end{aligned}
$$

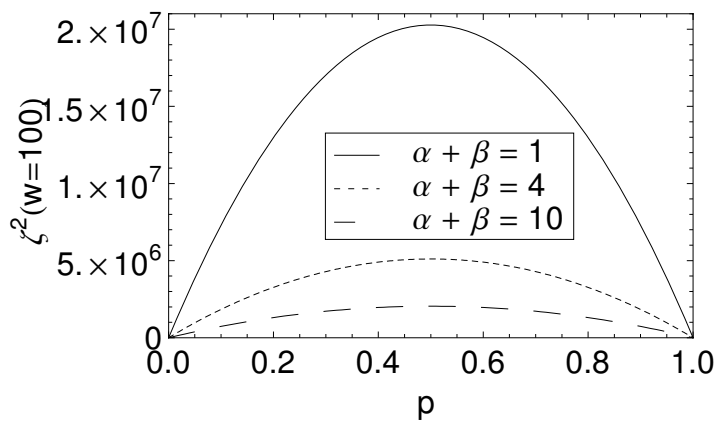

Fig. 4. Variance of sample mean as a function of $p$

$\zeta^{2}(w)$ approaches 0 as the window size increases. How quickly it tends to zero, depends on the sum $\alpha+\beta$ and also the relative difference between $\alpha$ and $\beta$. Processes with long time constants $(\alpha+\beta$ small $)$ increase the value of $\zeta^{2}(w)$ resulting in less accuracy. The burstiness of the sources can be described by the activity parameter $p=\frac{\alpha}{\alpha+\beta}$. Keeping $\alpha+\beta$ constant, $\zeta^{2}(w)$ reaches its maximum value when $p=0.5$. This is shown in Fig. 4 for a window size of $100 \mathrm{~s}$.

With the knowledge of the variance of the sample mean we now turn to a discussion of the accepting probability given that the system is in state $n_{\max }$. 
Accepting a flow in this state is considered an error and a false acceptance. To emphasize the state-dependency, this error probability is termed $q_{n_{\max }}$. It can be seen from (17), that with a given confidence level and $l$ fixed, the required window size will depend on the values of $\alpha$ and $\beta$. As $\alpha$ and $\beta$ increase, the required window size or critical region can be reduced. Also as the activity parameter $p$ approaches one a more accurate decision can be made.

As $n_{\max }$ increases, the impact of one single flow on the aggregate will be reduced. This causes again an increase in $q_{n_{\max }}$ as the difference between $n_{\max }$ and $n_{\max }+1$ becomes more and more negligible. To state this another way, the size of the critical region must be increased when the aggregation level goes up to keep the level of confidence at a certain value.

To check (17) we run a simple simulation with $n_{\max }=50$ flows with mean rate $\xi=32000 b p s$. An admission decision is made based on measurements of a complete measurement window. With $l=1$, Fig. [5] shows how $q_{n_{\max }}$ is reduced as the window size is increased. The simulated results follow closely what is theoretically predicted.

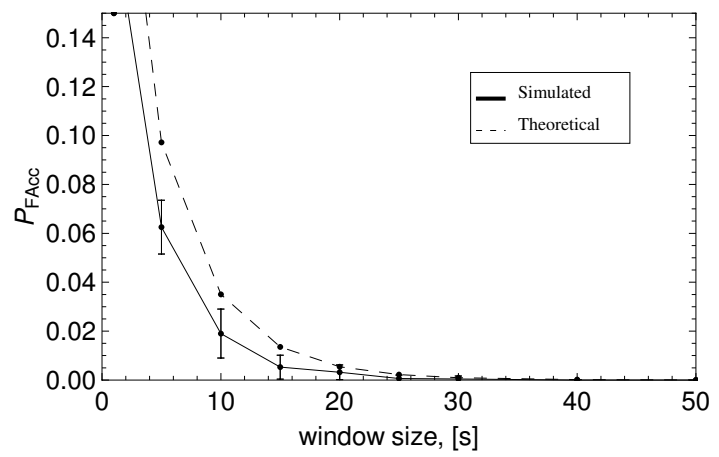

Fig. 5. Conditional probability of false acceptance in state $n_{\max }$

\section{Conclusion}

Measurement error is a critical aspect in measurement-based research [9]. However, there is in the literature of MBAC, unfortunately very limited work focusing on the impact of this error. An MBAC algorithm no matter how advanced, will be of little use if the measured parameters used by this algorithm include error of unknown quantity. This error causes uncertainty in the decision process and the degree of uncertainty abates with the length of the observation window. In this paper we have set up an analytical framework to analyze the measurement error. With analytical tractable sources with known covariance function, we are able to state the uncertainty of the decision process up front.

If on the other hand the covariance of the sources is unknown it must also be measured. Our work in progress extends this study to situations with only 
partial knowledge of the auto-covariance function. This work also includes the analysis of non-homogeneous flows.

The framework defined in this paper, considers the flow dynamics such that the influence of flow arrival and departure can be studied. A separate work will discuss this in more detail where attention also will be given to the effect of correlation at arrival points causing a cascade of false admissions. If the number of flows remains constant, increasing the window size will only improve performance. However, in reality this is not the case since flows may leave within a measurement window. Measuring too long will cause the number of connection to be changing at a rate causing instability in the measurements. With the help of simulations, errors not explained by the above reference system, can be revealed to get a more complete picture of the estimation errors. This current work sheds light on a more in-depth understanding of how measurement uncertainties and flow dynamics impact the admission decision in MBAC.

Acknowledgments. The helpful comments from anonymous referees are highly appreciated.

\section{References}

1. Knightly, E.W., Shroff, N.B.: Admission control for statistical QoS: Theory and practice. IEEE Network, 20-29 (March/April 1999)

2. Breslau, L., Jamin, S., Shenker, S.: Comments on the performance of measurementbased admission control algorithms. In: IEEE INFOCOM (2000)

3. Grossglauser, M., Tse, D.N.C.: A framework for robust measurement-based admission control. IEEE/ACM Trans. Networking 7(3), 293-309 (1999)

4. Moore, A.W.: Measurement-based management of network resources. Technical report, University of Cambridg, Cambridge CB3 OFD, United Kingdom (April 2002)

5. Grossglauser, M., Tse, D.N.C.: A time-scale decomposition approach to measurement-based admission control. IEEE/ACM Trans. Networking 11(4), 550-563 (2003)

6. Dziong, Z., Juda, M., Mason, L.G.: A framework for bandwidth management in ATM networks - aggregate equivalent bandwidth estimation approach. IEEE/ACM Trans. Networking 5(1), 134-147 (1997)

7. Key, P.B., Gibbens, R.J., Kelly, F.P.: A decision-theoretic approach to call admission control in atm networks. IEEE Journal on Selected Areas in Communications (JSAC) 13(6), 1101-1114 (1995)

8. Brockwell, P.J., Davis, R.A. (eds.): Introduction to Time Series and Forecasting, 2nd edn. Springer, Heidelberg (2002)

9. Krishnamurthy, B., Willinger, W.: What are our standards for validation of measurement-based networking research? Performance Evaluation Review 36(2), 64-68 (2008) 